\title{
RFID Signal Reconstruction of Internet of Things Based on Compressed Sensing
}

\author{
Li Fen ${ }^{1}$, Pan Xuefeng ${ }^{1}$, Meng Gang ${ }^{2}$
}

1.Department of computer science, Wuhan Qingchuan University, Wuhan, 430204, China

2.Change Jiang Institute of Survey, Planning, Design and Research, Wuhan , 430010, China

Keywords: Compressed sensing; signal reconstruction; Internet of things; information sampling; sparse matrix

\begin{abstract}
The basic theories of signal sampling is the famous Nyquist sampling theorem, Nyquist sampling theorem pointed out that the bandwidth of the signal sampling rate reached more than two times, in order to accurately reconstruct the original signal by the sampling signal. However, with the increasing demand for information, the bandwidth of the signal carrying information more and more wide, so naturally raises a question: whether to ensure no loss of information, the sampling signal with a much lower rate than the Nyquist sampling theorem, but also can fully recover the signal. This paper describes the basic theory and core issues of compressed sensing, and introduces compression based reconstruction algorithms: base pursuit algorithm (BP algorithm) and orthogonal matching pursuit algorithm (OMP algorithm). Secondly, the basic principle of sparse representation is analyzed, the main methods of sparse representation are introduced, and the sparse representation method based on Gabor dictionary and its application in compressed sensing is emphasized.
\end{abstract}

\section{Introduction}

After a few years of development, the theoretical basis of compressed sensing has been filled, and also carried out in-depth research on compressed sensing in all aspects. At present, the research of compressed sensing mainly includes the following aspects: sparse representation of signal, design of measurement matrix, reconstruction algorithm and compressed sensing application. The sparse representation of the signal is to project the signal to another space, so that most of the signal in the space is zero or very small, only a few of the signal values are dominant, and the resulting transformation vectors are sparse. This representation is a concise representation of the signal in another space, but it does not lose the information of the signal. The measurement matrix refers to the source signal is mapped into a low dimensional space projection matrix. The measurement matrix to satisfy the restricted isometry property (Restricted Isometry, Property, RIP), the measurement matrix through the source signal for global observation observed signal, finally using reconstruction algorithm recover the source signals from the observed signals in the measurement matrix and reconstruction algorithm, and how to design accurate and efficient compressed sensing is a hot research problem. The application of CS is currently a hot topic, such as analog information conversion (Analog to Information Converter, AIC, AIC) is the compressed sensing theory to practice can not avoid the problem of how to obtain the signal in the process of making the channel number less, high real-time and efficient structure reconstruction algorithm is the focus of current research in the front.

\section{Compressed Sensing}

Signal acquisition, coding and decoding process of traditional encoding: end first signal sampling, and of all the sampling value transform, and one of the important coefficient of amplitude and position encoding, finally encoding value storage or transmission, decoding process signal is simply the inverse process of encoding, the received signals are recovered by signal decompression, inverse transform. With this traditional encoding and decoding method, the sampling rate of the signal can not be less than 2 times of the signal bandwidth, which makes the hardware system face 
great pressure of sampling rate. In addition, in the process of compression coding, a small amount of small coefficients are discarded, resulting in waste of data calculation and memory resources.

Suppose there is a signal $f\left(f \in R^{N}\right)$, Count $\operatorname{Reg} N$, Base vector $\Psi_{i}(i=1,2, \ldots, N)$, The signal is transformed:

$$
f=\sum_{i=1}^{N} a_{i} \psi_{i} \text { or } f=\Psi \alpha(1)
$$

Obviously $f$ Is the signal in the time domain representation, $\alpha$ It's the signal $\Psi$ Representation of domain. Whether the signal has sparsity or approximate sparsity is the key problem of compressed sensing theory, if $(1-1)$ In the form of $\alpha$ only $K$ Nonzero values $(N>>K)$ The signal is sparse after sorting and exponentially decreasing and approaching zero. Sparse representation of signals is a priori condition for compressed sensing. The compressed sensing process can be divided into two steps under the premise that the known signal is compressible:

(1) design a not related to the transformation base $M \times N(M<<N)$ The dimension measurement matrix is used to observe the signal $M$ Measure vector of dimension.

By the $M$ Reconstruction signal of dimension measurement vector.

Not related to the transformation matrix $M \times N(M<<N)$ Measurement matrix $\phi$ The linear projection is obtained by linear projection of the $\operatorname{signal}^{y}$ :

$$
y=\phi f
$$

The measured value is one ${ }^{y}$ It's one $M$ Dimension vector, so that the measurement object comes from the vector $N$ Dimensionality reduction $M$ Dimension. The observation process is non adaptive, that is, the choice of the measurement matrix is less dependent on the signal $f$. Measurement matrix design requirements signal from $f$ Convert to $y$ In the process of measurement $K$ A measured value does not destroy the original signal information, to ensure accurate signal reconstruction.

Due to signal $f_{\text {It }}$ is sparse representation It can be expressed in sparse form, and the upper form can be expressed as the lower form:

$$
y=\phi f=\Psi \phi \alpha=\Theta \alpha
$$

among $\Theta$ It's one $M \times N$ Matrix. In the formula, the number of equations is much smaller than the unknown, the equation has no definite solution, unable to reconstruct the signal. However, due to the fact that the signal isKSparse, if the upper form $\Theta$ Satisfying finite isometry property(Restricted Isometry Property, AbbreviationRIP), That is, for any K sparse signal $f$ Sum constant $\delta_{k} \in(0,1)$, matrix $\Theta$ Satisfy:

$$
1-\delta_{k} \leq \frac{\|\Theta f\|_{2}^{2}}{\|f\|_{2}^{2}} \leq 1+\delta_{k}
$$

The $\mathrm{K}$ coefficients can be reconstructed accurately from $\mathrm{M}$ measurements. The equivalent condition of RIP property is the measurement matrix $\phi$ Sparse basis $\Psi$ Uncorrelated. At present, there are mainly kinds of measurement matrices used for Compressive Sensing: Gauss random matrix, two valued random matrix (Bernoulli matrix), Fu Liye random matrix, Hadamar matrix, uniform sphere matrix, etc.

\section{Signal Reconstruction}

The reconstruction algorithm and measurement matrix has a close relationship. In various reconstruction algorithms, the reconstruction quality of MP, OMP and ROMP is closely related to the measurement matrix, that is to say, the nonlinear correlation of the column vectors of the measurement matrix is the prerequisite for the reconstruction algorithm to select the column vectors. 
Therefore, if we can construct a good matrix, it will simplify the steps of the reconstruction algorithm greatly and improve the quality of signal reconstruction.

At present, the main measurement matrix is available:

(1) Gauss measurement matrix, each element in the matrix is independent of the mean value is 0 , the variance is $1 / \mathrm{M}$ ( $\mathrm{M}$ is the number of measurements) normal distribution.

(2) the Bernoulli measurement matrix, each element in the matrix obeys the symmetric Bernoulli distribution.

(3) Fu Liye random measurement matrix, $\mathrm{M}$ row vectors in the matrix obey uniform random distribution, and all column vectors are all normalized.

At present, the research of measurement matrix is mainly concerned:

(1) the measurement matrix transformation of the existing, the nature is more perfect, more conducive to the reconstruction algorithm.

(2) starting from an orthogonal matrix, deleting or increasing some of the rows or columns, obtaining a new measurement matrix, and then testing its performance in the application.

(3) according to the direction of an application, a nonrandom measurement matrix is constructed to satisfy the UUP characteristics and sparse signal reconstruction characteristics.

In the research of measurement matrix, an improvement of measurement matrix is given.

Because of the measurement matrix $\Phi$ The minimum singular value must be greater than a

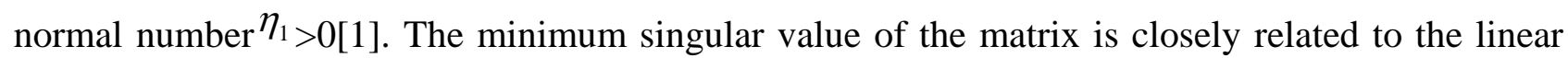
correlation property of the matrix. The greater the minimum singular value is, the stronger the independence of the matrix is, and when the minimum singular value tends to 0 , the independence of the matrix disappears. Therefore, the minimum singular value of the measurement matrix plays an important role, and a method is proposed to improve the minimum singular value of the matrix.

QR decomposition is a method of increasing the singular value of the matrix without changing the nature of the measurement matrix. And the standard QR decomposition compared to the following processing of measurement matrix: firstly the measurement matrix QR decomposition, obtained the upper triangular matrix R and Q matrix; R matrix to save the main diagonal elements, the position value is set to $0 \mathrm{NEW}$ momentsfront $\hat{R}$, A new measurement matrix is obtained $\hat{\Phi}$. The Gauss random matrix is taken as an example $\Phi$ Decomposition:

Where $\mathrm{Q}$ is a $\mathrm{N} * \mathrm{~N}$ matrix, and $\mathrm{R}$ is an upper triangular matrix of $\mathrm{N} * \mathrm{M}$ :

$$
\Phi^{T}=Q R
$$

$$
\Phi=R^{T} Q^{T}
$$

Because the element values on the main diagonal of matrix $\mathrm{R}$ are much larger than those on non diagonal elements, only the diagonal elements of $\mathrm{R}$ are preserved, and the rest elements are set to 0 , and the new diagonal matrix is obtained $\hat{R}$ Thus a new one is obtained $\hat{\Phi}$ :

$$
\hat{\Phi}=\hat{R}^{T} \bullet Q^{T}
$$

$\hat{\Phi}$ Satisfying the characteristics of measurement matrix, $\hat{\Phi}$ Is the minimum singular value greater than that of the least singular value? $\Phi$ Minimum singular value, $\hat{\Phi}$ Is the maximum singular value smaller than that of the singular value? $\Phi$ The maximum singular value.

\section{Experiment}

By analyzing the reconstruction errors of different $\mathrm{M} / \mathrm{N}$ and different $\mathrm{K}$, the influence of the number of observations and sparsity on the performance of signal compression reconstruction is investigated. A self-designed signal with a fixed length of $\mathrm{N}=48$ is used to remove the values shown by $\mathrm{M}$ and $\mathrm{K}$, and the OMP algorithm is used to reconstruct the designed signals: error $=$ $\|x-\hat{x}\|^{2} /\|x\|^{2}$ However, the reconstruction error in BP algorithm is very small, and the error in M with different sparsity $\mathrm{K}$ and different observation varies greatly, so it is not suitable to use reconstruction error in the analysis, and the direct use of signal-to-noise ratio is reasonable. When 
the signal length $\mathrm{N}$ is small, the reconstruction effect of BP algorithm is better. When the value of $\mathrm{N}=120$ is obtained, the following set of data is obtained:

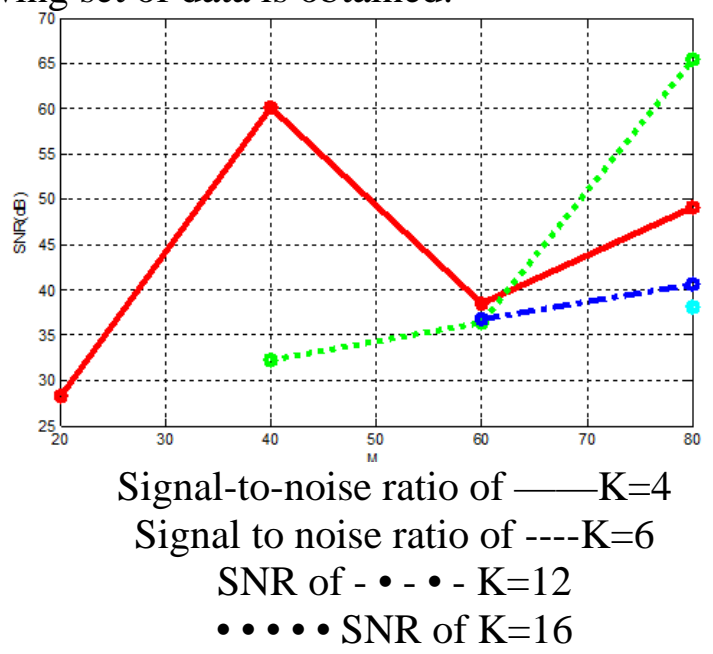

Figure 1 reconstructed signal to noise ratio using BP reconstruction

\section{Summary}

In the aspect of reconstruction algorithm, the classical OMP algorithm is analyzed and introduced, and the algorithm description and experimental results are given. The framework of BP algorithm is applied to sparse signal compressed sensing, and the sparse BP algorithm and OMP algorithm are studied in detail, and the signal reconstruction is programmed. The experimental results show that the compressed sensing algorithm using the BP framework has higher accuracy than the traditional OMP algorithm, and the reconstruction error is small, and the reconstructed signal noise is relatively high, so it is an algorithm worthy of everyone's application.

\section{Acknowledgement}

Basic Theory and Key Technology of Massive Data Management Supporting Technology Innovation

\section{Reference}

[1] Weisen Pan, Shizhan Chen, ZhiyongFeng. Investigating the Collaborative Intention and Semantic Structure among Co-occurring Tags using Graph Theory. 2012 International Enterprise Distributed Object Computing Conference, IEEE, Beijing, pp. 190-195.

[2] Jennifer W. Chan, Yingyue Zhang, and Kathryn E. Uhrich, Amphiphilic Macromolecule Self-Assembled Monolayers Suppress Smooth Muscle Cell Proliferation, Bioconjugate Chemistry, 2015, 26(7), 1359-1369.

[3] Yingyue Zhang, Evan Mintzer, and Kathryn E. Uhrich, Synthesis and Characterization of PEGylatedBolaamphiphiles with Enhanced Retention in Liposomes, Journal of Colloid and Interface Science, 2016, 482, 19-26.

[4] Jonathan J. Faig, AlyshaMoretti, Laurie B. Joseph, Yingyue Zhang, Mary Joy Nova, Kervin Smith, and Kathryn E. Uhrich, Biodegradable Kojic Acid-Based Polymers: Controlled Delivery of Bioactives for Melanogenesis Inhibition, Biomacromolecules, 2017, 18(2), 363-373. 